\title{
Development and Physicochemical Characterization of Acetalated Dextran Aerosol Particle Systems for Deep Lung Delivery
}

\author{
Zimeng Wang ${ }^{1}$, Sweta K. Gupta ${ }^{1}$, Samantha A. Meenach ${ }^{1,2}$ \\ ${ }^{1}$ University of Rhode Island, College of Engineering, Department of Chemical \\ Engineering, Kingston, RI 02881, USA \\ ${ }^{2}$ University of Rhode Island, College of Pharmacy, Department of Biomedical and \\ Pharmaceutical Sciences, Kingston, RI 02881, USA
}

Corresponding Author: Samantha A. Meenach, University of Rhode Island, 205 Crawford Hall, 16 Greenhouse Road, Kingston, RI, 02881, USA. Email: smeenach@uri.edu 


\section{ABSTRACT}

Biocompatible, biodegradable polymers are commonly used as excipients to improve the drug delivery properties of aerosol formulations, in which acetalated dextran (Ac-Dex) exhibits promising potential as a polymer in various therapeutic applications. Despite this promise, there is no comprehensive study on the use of Ac-Dex as an excipient for dry powder aerosol formulations. In this study, we developed and characterized pulmonary drug delivery aerosol microparticle systems based on spraydried Ac-Dex with capabilities of (1) delivering therapeutics to the deep lung, (2) targeting the particles to a desired location within the lungs, and (3) releasing the therapeutics in a controlled fashion. Two types of Ac-Dex, with either rapid or slow degradation rates, were synthesized. Nanocomposite microparticle (nCmP) and microparticle (MP) systems were successfully formulated using both kinds of Ac-Dex as excipients and curcumin as a model drug. The resulting MP were collapsed spheres approximately $1 \mu \mathrm{m}$ in diameter, while the $\mathrm{nCmP}$ were similar in size with wrinkled surfaces, and these systems dissociated into $200 \mathrm{~nm}$ nanoparticles upon reconstitution in water. The drug release rates of the Ac-Dex particles were tuned by modifying the particle size and ratio of fast to slow degrading Ac-Dex. The $\mathrm{pH}$ of the environment was also a significant factor that influenced the drug release rate. All $\mathrm{nCmP}$ and $\mathrm{MP}$ systems exhibited desirable aerodynamic diameters that are suitable for deep lung delivery (e.g. below $5 \mu \mathrm{m}$ ). Overall, the engineered Ac-Dex aerosol particle systems have the potential to provide targeted and effective delivery of therapeutics into the deep lung. 
KEYWORDS: Acetalated dextran, nanocomposite microparticles, microparticles, pulmonary delivery, spray drying, controlled release

\section{INTRODUCTION}

Pulmonary drug delivery has exhibited promising potential in the treatment of lung diseases, as it allows for the delivery of a wide range of therapeutics directly and efficiently to the lungs, thereby increasing local drug concentration, reducing systemic side effects, providing a rapid onset of pharmaceutical action, and avoiding the first-pass metabolism associated with the liver (Belotti et al., 2015; Cui et al., 2011; Mansour et al., 2009; Meenach et al., 2012). The deep lung (alveolar) region can be utilized as a route for systematic drug delivery due to the enormous surface area available and nearby plentiful capillary vessels that facilitate drug absorption, the very thin (approximately 0.1 $\mu \mathrm{m})$ liquid layer over the alveoli that ensures rapid and unhindered drug absorption, and low enzymatic activity, which enhances drug availability (Collier et al., 2016; Cui et al., 2011; Hoang et al., 2014). As a result, various therapeutics such as antibiotics, proteins, peptides, anti-cancer drugs (Wu et al., 2014), plasmid DNA (Takashima et al., 2007), siRNA (Jensen et al., 2010), and anti-tuberculosis (TB) drugs have been employed in inhalation formulations for the treatment of pulmonary diseases such as asthma, chronic obstructive pulmonary disease (COPD), cystic fibrosis (CF)-related pulmonary infections, and lung cancer (Meenach et al., 2013a; Wu et al., 2014).

Dry powders are a dosage formulation that delivers therapeutics to the lung, in the form of particles, using a dry powder inhaler (Wu et al., 2014). Compared with liquid aerosols, these formulations offer additional benefits such as enhanced stability of the 
formulation, controllable particle size for targeting different regions of the lung, and increased drug loading of hydrophobic payloads (Cohen et al., 2010; Meenach et al., 2013a). Spray drying has proven to be a suitable technology in the preparation of dry powder therapeutics (Meenach et al., 2013a), as it is capable of producing respirable microparticles for deep lung delivery with acceptable aerosol dispersion properties (Belotti et al., 2015). Properties of dry powder particles such as particle size, particle shape, and surface morphology can be modified by controlling the spray drying production process, thus providing desirable particle characteristics (Belotti et al., 2015; Wu et al., 2014).

Biocompatible, biodegradable polymers such as poly(e-caprolactone) (PCL) and poly(lactic-co-glycolic acid) (PLGA) have been used as dry powder formulation excipients to carry drug molecules, protect drugs from degradation, and impart sustained release to aerosol formulations (Mansour et al., 2009). However, PLGA and PCL delivery systems show significant burst release of their payloads due to bulk erosion of the polymers and it is difficult to control the polymer degradation rate and modulate their release profiles (Ulery et al., 2011). Acetalated dextran (Ac-Dex) is an acid-sensitive, biodegradable, biocompatible polymer prepared via a one-step reaction by reversibly modifying dextran with acetal groups. This modification reverses the solubility properties of dextran from hydrophilic to hydrophobic, making it possible to form polymeric particles using standard emulsion or nanoprecipitation techniques. In contrast to other commonly used polymers such as PLGA, polylactic acid, and PCL, Ac-Dex exhibits attractive properties suitable for the controlled release of therapeutic payloads. By controlling the reaction time during the formation of Ac-Dex, the ratio of cyclic acetal 
groups (with a slower degradation rate) to acyclic acetal groups (with a faster degradation rate) can be adjusted. As a result, the degradation rate of the resulting Ac-Dex can be tuned from hours to months to suit various applications. Moreover, the acid-sensitivity of Ac-Dex enables it to degrade faster in lower $\mathrm{pH}$ environments, such as lysosomes in macrophage or tumor cells, allowing for controlled release of drug within these cells. Furthermore, Ac-Dex degrades into neutral by-products, which avoids undesirable changes in the micro-environmental $\mathrm{pH}$ in the body. Finally, Ac-Dex offers the potential of targeted delivery, due to the presence of dextran chains that can be further modified with a variety of functional targeting moieties (Bachelder et al., 2008; Broaders et al., 2009; Kauffman et al., 2012).

Owing to the aforementioned advantages, Ac-Dex has been widely applied in the formation of polymeric carriers for drug delivery. Porous Ac-Dex microparticles loaded with the chemotherapeutic camptothecin were developed for pulmonary delivery using emulsion techniques. These systems exhibited a respirable fraction of $37 \%$ and experimental mass mean aerodynamic diameters from 5.3 - $11.9 \mu \mathrm{m}$ (Meenach et al., 2012). Ac-Dex nanoparticle systems have been investigated in the application of protein delivery for immunotherapy (Broaders et al., 2009), gene delivery to phagocytic and nonphagocytic cells (Cohen et al., 2010), tandem delivery of peptide and chemotherapeutic for controlled combination chemotherapy (Cui et al., 2011), delivery of the host-mediated compound AR-12 (Arno Therapeutics; formerly known as OSU-03012) for the treatment of Leishmania donovani (Collier et al., 2016), and the control of Salmonella infections (Collier et al., 2016). Both Ac-Dex nanoparticles and microparticles loaded with 
horseradish peroxidase have been evaluated to improve vaccine stability outside cold chain conditions (Kanthamneni et al., 2012).

Despite this work, there is no comprehensive study on using Ac-Dex as an excipient for dry powder aerosol formulations produced via spray drying. In this study, we aimed to develop and characterize dry powder pulmonary delivery systems based on spray-dried Ac-Dex particles with capabilities of (a) delivering therapeutics to the deep lung, (b) targeting the particles to a particular location within the lungs, and (c) releasing therapeutics at a controlled rate. Previous studies have shown that: (a) aerodynamic diameter $\left(d_{a}\right)$ determines the region of the lungs where particles will deposit, where particles with an $d_{a}$ of $1-5 \mu \mathrm{m}$ tend to deposit in the deep lung region (Meenach et al., 2013a); (b) geometric size plays an important role in the cellular uptake of particles, where nanoscale particles (approximately $150 \mathrm{~nm}$ ) tend to escape phagocytic uptake (He et al., 2010), while particles larger than $1 \mu \mathrm{m}$ will suffer from macrophage clearance in the alveoli (Kho et al., 2010); and (c) the drug release rate of Ac-Dex particles can be tuned by modifying the synthesis time of the Ac-Dex polymer (Kauffman et al., 2012; Meenach et al., 2012).

To prepare the engineered particle systems, two types of Ac-Dex with rapid or slow degradation rates were synthesized. Nanocomposite microparticle $(\mathrm{nCmP})$ and microparticle (MP) systems were formulated using both kinds of Ac-Dex as the excipient. Curcumin was used as model drug owing to its high hydrophobicity (similar to many other pulmonary therapeutics) and fluorescence (allowing for easy detection). The $\mathrm{nCmP}$ were prepared by spray drying an aqueous suspension of CUR-loaded Ac-Dex nanoparticles (NP, $200 \mathrm{~nm}$ ) and the solid MP were formulated by spray drying a solution 
of Ac-Dex and CUR in a solution of tetrahydrofuran (THF) and acetone. We hypothesize that upon pulmonary administration, the $\mathrm{nCmP}$ will deposit in the deep lung, decompose into free NP, and facilitate the sustained release of drug to the targeted site, while the MP will remain the original size after deposition in the deep lung region. A schematic of the described particle preparation and design is shown in Figure 1. Overall, the goal of the described research was the initial development and physicochemical characterization of dry powder Ac-Dex aerosol particle systems with the potential for effective delivery of therapeutics.

\section{MATERIALS AND METHODS}

\subsection{Materials}

Dextran from Leuconostoc mesenteroides (9000-11000 MW), pyridinium ptoluenesulfonate (PPTS, 98\%), 2-methoxypropene (2-MOP, 97\%), triethylamine (TEA, $\geq$ 99\%), anhydrous dimethyl sulfoxide (DMSO, $\geq 99.9 \%$ ), poly(vinyl alcohol) (PVA, MW 13,000-23,000, 87-89\% hydrolyzed), dichloromethane (DCM, anhydrous, $\geq 99.8 \%$ ), deuterium chloride (DCl, 35 weight $\%$ in $\mathrm{D}_{2} \mathrm{O}, 99$ atom $\left.\% \mathrm{D}\right)$, Tween ${ }^{\circledR} 80$, curcumin, sodium acetate $(\geq 99 \%)$, acetic acid solution $(1.0 \mathrm{~N})$, acetone $(\geq 99.8 \%)$, tetrahydrofuran (THF, $\geq 99 \%$ ), and methanol (anhydrous, $\geq 99.9 \%$ ) were obtained from Sigma-Aldrich (St. Louis, MO). Deuterium oxide $\left(\mathrm{D}_{2} \mathrm{O}, 99.8 \%\right.$ atom $\left.\mathrm{D}\right)$ was obtained from Acros Organics (Geel, Belgium). Phosphate buffered saline (PBS) was obtained from Fisher Scientific (Somerville, NJ). Hydranal ${ }^{\circledR} \mathrm{KF}$ reagent was obtained from Fluka Analytical. 


\subsection{Synthesis and NMR Analysis of Acetalated Dextran (Ac-Dex)}

Ac-Dex was synthesized as described previously (Bachelder et al., 2008) with minor modifications. $1 \mathrm{~g}$ of lyophilized dextran and $25 \mathrm{mg}$ of PPTS were dissolved in 10 $\mathrm{mL}$ of anhydrous DMSO. The resulting solution was reacted with $5 \mathrm{~mL}$ of 2-MOP under nitrogen gas for 5 minutes to prepare Ac-Dex with a rapid degradation rate (Ac-Dex5min) or for 3 hours to prepare Ac-Dex with a slower degradation rate (Ac-Dex-3h). The reaction was quenched with $1 \mathrm{~mL}$ of TEA. The reaction mixture was then precipitated in basic water (water and TEA, $\mathrm{pH}$ 9), vacuum filtered, and lyophilized $\left(-50{ }^{\circ} \mathrm{C}, 0.023\right.$ mbar) for 24 hours to yield a solid product.

The cyclic-to-acyclic (CAC) ratio of acetal coverage and degrees of total acetal coverage per 100 glucose molecules was confirmed by ${ }^{1} \mathrm{H}$ NMR spectroscopy (Bruker $300 \mathrm{MHz}$ NMR, MA). $10 \mathrm{mg}$ of Ac-Dex was added to $700 \mu \mathrm{L}$ of $\mathrm{D}_{2} \mathrm{O}$ and was hydrolyzed with $30 \mu \mathrm{L}$ of $\mathrm{DCl}$ prior to analysis. The hydrolysis of one cyclic acetal group produces one acetone molecule whereas one acyclic acetal produces one acetone and one methanol molecule each. Consequently, from the normalized integration of peaks related to acetone, methanol, and the carbon ring of dextran, the CAC ratio of acetal coverage and degrees of total acetal coverage per 100 glucoses were determined.

\subsection{Formation of CUR-Loaded Ac-Dex Nanoparticles (CUR NP)}

Curcumin-loaded nanoparticles (CUR NP) were prepared via an oil/water emulsion solvent evaporation using Ac-Dex-5min, Ac-Dex-3h, or a mixture of both types of Ac-Dex (50\% w/w). $49 \mathrm{mg}$ of Ac-Dex and $1 \mathrm{mg}$ of CUR were dissolved in $1 \mathrm{~mL}$ of DCM over an ice bath, establishing the organic phase. The aqueous phase was comprised 
of $6 \mathrm{~mL}$ of $3 \%$ PVA in PBS and was added to the organic phase. The resulting mixture was sonicated (Q500 Sonicator, Qsonica, Newtown, CT) for 30 seconds with a 1 second on/off pulse at $70 \%$ amplitude. The emulsion was transferred to a spinning solution of 0.3\% PVA in PBS and was stirred for 3 hours to allow for evaporation of the organic solvent and particle hardening. The solution was then centrifuged at $19802 \times \mathrm{g}$ for 20 minutes to collect the nanoparticles. Nanoparticles were washed once with DI water, redispersed in $0.1 \%$ PVA, and lyophilized $\left(-50{ }^{\circ} \mathrm{C}, 0.023 \mathrm{mbar}\right)$ for 48 hours. The resulting NP systems were: CUR NP-5min (made of Ac-Dex-5min only), CUR NP-3h (made of Ac-Dex-3h only), and CUR NP-h (50 wt\% Ac-Dex-5min and 50 wt\% Ac-Dex$3 h)$.

\subsection{Formulation of CUR Nanocomposite Microparticles (CUR nCmP) via Spray}

\section{Drying}

CUR nCmP were prepared via the spray drying of an aqueous suspension of each type of CUR NP $(0.5 \%$, w/v) using a Büchi B-290 spray dryer (Büchi Labortechnik, AG, Switzerland) in open mode. The CUR NP suspension was sonicated for 10 minutes before spray drying. The spray drying conditions were as follows: inlet temperature of 60 ${ }^{\circ} \mathrm{C}$ (outlet temperature of $32 \pm 2{ }^{\circ} \mathrm{C}$ ), $0.7 \mathrm{~mm}$ nozzle diameter, atomization gas flow rate of $414 \mathrm{~L} / \mathrm{h}$ using dry nitrogen, aspiration rate of $28 \mathrm{~m}^{3} / \mathrm{h}$, pump rate of $0.9 \mathrm{~mL} / \mathrm{min}$, and nozzle cleaner rate of 3 . The resulting $\mathrm{nCmP}$ were separated in a high-performance cyclone, dried for 15 minutes in the spray dryer for further removal of residual water, collected in a sample collector, and stored in amber glass vials in a desiccator at $-20^{\circ} \mathrm{C}$. 
$\mathrm{nCmP}$ comprised of each kind of NP described previously were produced: $\mathrm{nCmP}-5 \mathrm{~min}$, $\mathrm{nCmP}-3 \mathrm{~h}$, and nCmP-h, correspondingly.

\subsection{Formulation of CUR Microparticles (CUR MP) via Spray Drying}

Solid curcumin-loaded microparticles (CUR MP) were prepared via the spray drying of an organic solution comprised of Ac-Dex and CUR using a Büchi B-290 spray dryer in closed mode. The organic solutions were prepared by dissolving CUR and AcDex $(2: 98 \mathrm{w} / \mathrm{w})$ in an organic solvent comprised of $85 \%$ acetone and $15 \% \mathrm{THF}(\mathrm{v} / \mathrm{v})$ at a solids concentration of $2 \%(\mathrm{w} / \mathrm{v})$. The spray drying conditions were as follows: inlet temperature of $60{ }^{\circ} \mathrm{C}$ (outlet temperature of $40 \pm 2{ }^{\circ} \mathrm{C}$ ), $0.7 \mathrm{~mm}$ nozzle diameter, atomization gas flow rate of $414 \mathrm{~L} / \mathrm{h}$ using UHP dry nitrogen, aspiration rate of $40 \mathrm{~m}^{3} / \mathrm{h}$, pump rate of $3 \mathrm{~mL} / \mathrm{min}$, and nozzle cleaner rate of 0 . The resulting MP were separated in a high-performance cyclone, dried for 15 minutes in the spray dryer for further removal of residual solvent, collected in a sample collector, and stored in amber glass vials in a desiccator at $-20^{\circ} \mathrm{C}$. The resulting MP were: MP-5min (from Ac-Dex-5min), MP-3h (from Ac-Dex-3h), and MP-h (from $50 \mathrm{wt} \%$ Ac-Dex-5min and $50 \mathrm{wt} \%$ Ac-Dex-3h).

\subsection{Particle Size, Size Distribution, and Zeta Potential Analysis}

The average diameter, size distribution, and zeta potential of the original NP and the NP released from the dispersion of $\mathrm{nCmP}$ in water were measured by dynamic light scattering (DLS) using a Malvern Nano Zetasizer (Malvern Instruments, Worcestershire, $\mathrm{UK})$. The original $\mathrm{NP}$ and $\mathrm{nCmP}$ were dispersed in $\mathrm{DI}$ water $(\mathrm{pH}=7,0.3 \mathrm{mg} / \mathrm{mL})$ prior 
to analysis. All experiments were performed in triplicate with a scattering angle of $173^{\circ}$ at $25^{\circ} \mathrm{C}$.

\subsection{Particle Morphology and Shape Analysis via Scanning Electron Microscopy} (SEM)

The shape and surface morphology of the $\mathrm{nCmP}$ and $\mathrm{MP}$ were evaluated by SEM using a Zeiss SIGMA VP Field Emission-Scanning Electron Microscope (FE-SEM) (Germany). Particle samples were placed on aluminum SEM stubs (Ted Pella, Inc.,

Redding, CA) with double-sided adhesive carbon tabs. The samples were coated with a thin film of a gold/palladium alloy using a BIO-RAD sputter coating system at $20 \mu \mathrm{A}$ for 60 seconds under argon gas. Images were captured at $8 \mathrm{kV}$ at various magnifications. The geometric mean diameter and standard deviation of the MP were measured digitally from SEM images using ImageJ software (Rasband, 1997-2016). Representative micrographs (5000x magnification) for each sample were analyzed by measuring the diameter of at least 300 particles.

\subsection{Tapped Density Evaluation of $\mathrm{nCmP}$ and MP}

The tapped density of the particles was measured as described previously with minor modifications (Tomoda et al., 2008). 35 - $40 \mathrm{mg}$ of particles was weighed into a glass tube. The tube was tapped 200 times to ensure efficient packing of the particles and then the volume occupied by the particles was measured using calipers. The density of the particles was then determined by the following equation: 
$\rho=\frac{m}{V}$

where $\rho$ is the tapped density, $\mathrm{m}$ is the particle mass, and $\mathrm{V}$ is the volume occupied by the particles as determined by measuring the height of the particles in the tube with a known diameter $(5 \mathrm{~mm})$. The theoretical mass median aerodynamic diameter $\left(\mathrm{MMAD}_{\mathrm{T}}\right)$ of the particles was then calculated using the following equation:

$\operatorname{MMAD}_{\mathrm{T}}=d \sqrt{\frac{\rho}{\rho^{*}}}$

where $\mathrm{d}$ is the geometric diameter determined by ImageJ, $\rho$ is the tapped density of the particles, and $\rho^{*}=1 \mathrm{~g} / \mathrm{cm}^{3}$, which is the reference density of solid polymer.

\subsection{Drug Loading Analysis of $\mathrm{nCmP}$ and MP}

The drug loading and encapsulation efficiency of CUR nCmP and CUR MP were determined via fluorescence spectroscopy (Biotek Cytation 3, Winooski, VT). All particle samples were dissolved in DMSO and were evaluated at $420 \mathrm{~nm}$ (excitation) and $520 \mathrm{~nm}$ (emission). The CUR drug loading and encapsulation efficiency (EE) of the particles were determined by the following equations:

Drug loading $=\frac{\text { mass of CUR loaded in particles }}{\text { mass of particles }}$

Encapsulation efficiency $(\mathrm{EE})=\frac{\text { mass of CUR loaded in particles }}{\text { initial mass of CUR in particles }} \times 100 \%$ 


\subsection{In Vitro Drug Release from $\mathrm{nCmP}$ and $\mathrm{MP}$}

The in vitro release profiles of CUR from $\mathrm{nCmP}$ and MP were determined via the release of suspended particles $(0.5 \mathrm{mg} / \mathrm{mL}, 1.5 \mathrm{~mL})$ in modified phosphate buffer $(0.1 \mathrm{M}$, $\mathrm{pH}=7.4,0.5 \mathrm{wt} \%$ Tween $\left.^{\circledR} 80\right)$ and modified acetate buffer $(0.1 \mathrm{M}, \mathrm{pH}=5,0.5 \mathrm{wt} \%$ Tween ${ }^{\circledR} 80$ ). The particle suspensions were incubated at $37^{\circ} \mathrm{C}$ and $100 \mathrm{rpm}$ (Digital Heat Block and ORBi shaker, Benchmark Scientific, Edison, NJ). At various time points, particle samples were centrifuged at $23102 \times \mathrm{g}$ for 5 minutes at $4{ }^{\circ} \mathrm{C}$ to isolate the NP. $200 \mu \mathrm{L}$ of supernatant was withdrawn and replaced by the same amount of fresh modified buffer in each sample. The withdrawn solutions were mixed with an equal volume of DMSO and analyzed for CUR content via fluorescence spectroscopy using the same method described for drug loading. The release data was fitted to several commonly utilized drug release models (Supplemental Information Section S.1) to elucidate the mechanism of drug release of Ac-Dex particles. The coefficient of determination $\left(\mathrm{R}^{2}\right)$ was applied to test the applicability of the described release models.

\subsection{Differential Scanning Calorimetry (DSC)}

The thermal phase transitions of $\mathrm{nCmP}, \mathrm{MP}$, and their raw components were determined via DSC using a TA Q10 DSC system (TA Instruments, New Castle, DE, USA) equipped with an automated computer-controlled TA instruments DSC refrigerated cooling system. 1 - $3 \mathrm{mg}$ of sample was weighed into Tzero ${ }^{\mathrm{TM}}$ alodine-coated aluminum pans that were hermetically sealed. The sealed pans were placed into the DSC furnace 
along with an empty sealed reference pan. The heating range was $0-200{ }^{\circ} \mathrm{C}$ at a heating rate of $10{ }^{\circ} \mathrm{C} / \mathrm{min}$.

\subsection{Powder X-Ray Diffraction (PXRD)}

The crystalline states of $\mathrm{nCmP}, \mathrm{MP}$, and its raw components were examined by PXRD using a Rigaku Multiflex X-ray diffractometer (The Woodlands, TX) with a $\mathrm{Cu}$ $\mathrm{K} \alpha$ radiation source $(40 \mathrm{kV}, 44 \mathrm{~mA})$. The samples were placed on a horizontal quartz glass sample holder $(3 \mathrm{~mm})$ prior to analysis. The scan range was $5-60^{\circ}$ in $2 \Theta$ with a step width of 0.1 and scan rate of $1 \% \mathrm{~min}$.

\subsection{Karl Fischer Coulometric Titration}

The water content of nCmP and MP was quantified by Karl Fischer (KF) titration using a $737 \mathrm{KF}$ coulometer (Metrohm, Riverview, FL). $5 \mathrm{mg}$ of powder was dissolved in anhydrous methanol. The resulting solution was injected into the KF reaction cell filled with Hydranal ${ }^{\circledR} \mathrm{KF}$ reagent and then the amount of water was analyzed. Pure solvent was also injected for use as a background sample.

\subsection{In Vitro Aerosol Dispersion Performance with the Next Generation Impactor (NGI)}

In vitro aerosol dispersion performance of $\mathrm{nCmP}$ and $\mathrm{MP}$ was evaluated using a Next Generation Impactor ${ }^{\mathrm{TM}}$ (NGI ${ }^{\mathrm{TM}}$, MSP Corporation, Shoreview, MN) equipped with a stainless steel induction port (USP throat adaptor) attachment and stainless steel gravimetric insert cups. The NGI ${ }^{\mathrm{TM}}$ was coupled with a Copley TPK 2000 critical flow 
controller, which was connected to a Copley HCP5 vacuum pump (Copley Scientific, United Kingdom). The air flow rate (Q) was measured and adjusted to $60 \mathrm{~L} / \mathrm{min}$ before each experiment in order to model the flow rate in a healthy adult lung. Glass fiber filters (55 mm, Type A/E, Pall Life Sciences, PA) were placed in the gravimetric insert cups for stages 1 through 7 to minimize particle bounce or re-entrapment (Meenach et al., 2013a) and these filters were weighed before and after the experiment to determine the particle mass deposited on each stage. Approximately $10 \mathrm{mg}$ of powder was loaded into a hydroxypropyl methylcellulose (HPMC, size 3, Quali- ${ }^{\circledR}$, Qualicaps ${ }^{\circledR}$ Inc., Whitsett, NC, USA) capsule and the capsule was placed into a human dry powder inhaler device (HandiHaler, Boehringer Ingelheim Pharmaceuticals, CT) attached to a customized rubber mouthpiece connected to the NGI ${ }^{\mathrm{TM}}$. Three HPMC capsules were loaded and released in each measurement and experiments were performed in triplicate. The NGITM was run with a delay time of $10 \mathrm{~s}$ and running time of $10 \mathrm{~s}$. For $\mathrm{Q}=60 \mathrm{~L} / \mathrm{min}$, the effective cutoff diameters for each stage of the impactor were given from the manufacturer as: stage $1(8.06 \mu \mathrm{m})$; stage $2(4.46 \mu \mathrm{m})$; stage $3(2.82 \mu \mathrm{m})$; stage $4(1.66$ $\mu \mathrm{m})$; stage $5(0.94 \mu \mathrm{m})$; stage $6(0.55 \mu \mathrm{m})$; and stage $7(0.34 \mu \mathrm{m})$. Our previous study on the relationship between particle mass distribution and payload distribution showed that no significant difference existed between the drug amount and particle mass in each chamber of NGI ( $p>0.05)$, indicating that the drug was uniformly dispersed in both CUR-MP and CUR-nCmP (Figure S1). The fine particle fraction (FPF), respirable fraction $(\mathrm{RF})$, and emitted dose (ED) were calculated as follows: 
Fine particle fraction $(\mathrm{FPF})=\frac{\text { mass of particles on Stages } 2 \text { through } 7}{\text { initial particle mass loaded into capsules }} \times 100 \%$

Respirable fraction $(\mathrm{RF})=\frac{\text { mass of particles on Stages } 2 \text { through } 7}{\text { total particle mass on all stages }} \times 100 \%$

Emitted dose $(E D)=\frac{\text { initial mass in capsules }- \text { final mass remaining in capsules }}{\text { initial mass in capsules }} \times 100 \%$

The experimental mass median aerodynamic diameter $(\mathrm{MMAD} E)$ and geometric standard deviation (GSD) for the particles were determined using a Mathematica ${ }^{\circledR}$ program written by Dr. Warren Finlay (Meenach et al., 2013a; W, 2008).

\subsection{Statistical Analysis}

All measurements were performed in at least triplicate. Values are given in the form of mean \pm standard deviation. The statistical significance of the results was determined using analysis of variance (ANOVA) and student's t-test. A p-value of $<0.05$ was considered statistically significant.

\section{RESULTS AND DISCUSSION}

\subsection{Preparation and Characterization of Ac-Dex and Curcumin Nanoparticles}

\subsubsection{NMR Analysis of Ac-Dex}

Successful synthesis of Ac-Dex was confirmed by ${ }^{1} \mathrm{H}$ NMR (Figure S2). Ac-Dex5min exhibited $61.2 \%$ cyclic acetal coverage (CAC) and $71.6 \%$ total conversion of $-\mathrm{OH}$ 
groups, while Ac-Dex-3h exhibited $82.5 \% \mathrm{CAC}$ and $80.0 \%$ total conversion of $-\mathrm{OH}$ groups, which matched our previous results (Wang et al., 2016; Wang and Meenach, 2016). The Ac-Dex with longer synthesis time (Ac-Dex-3h) exhibited a higher CAC, which was in accordance with previous studies. An increase in CAC is known to decrease polymer degradation and ultimately, the drug release rate, due to the slower degradation of the cyclic acetal groups on the Ac-Dex backbone (Bachelder et al., 2008; Broaders et al., 2009). Ac-Dex-3h also showed a higher total conversion of -OH groups, which could be a result the longer reaction time. This higher total acetal coverage is favorable in the enhancement of the stability of the PVA coating of nanoparticles (data not shown), thus ensuring small particle size and narrow size distribution.

\subsubsection{Dynamic Light Scattering (DLS) Analysis of Original and Redispersed CUR NP}

Average nanoparticle size, size distribution/polydispersion index (PDI), and zeta potential are shown in Table 1. No significant changes in NP size, PDI, or zeta potential was found between the original and redispersed NP $(p<0.05)$, indicating that the CUR NP maintained their properties after redispersion. The original and redispersed NP exhibited an average diameter of approximately $200 \mathrm{~nm}$, which is in the desirable range to avoid macrophage clearance and mucus entrapment (Kho et al., 2010). The low PDI value denotes a narrow size distribution of the NP, and the slightly negatively charged surface of nanoparticles, as measured by zeta potential, is desirable in order to reduce the interactions with negatively charged mucin fibers present in airway mucus (Lai et al., 2009). According to our preliminary experiments (data not shown), a low total conversion of -OH groups on the Ac-Dex results in NP with larger sizes and PDI due to 
NP agglomeration. This phenomenon could be a result of the reduced hydrophobicity of Ac-Dex with fewer -OH groups converted to acetal groups, which leads to insufficient absorption of PVA on the NP surface. However, the Ac-Dex in this study was prepared to produce NP with small sizes and low PDI, as the total conversion of -OH groups was kept in a higher range to prevent NP agglomeration.

\subsection{Preparation and Characterization of Nanocomposite Microparticles (nCmP) and Microparticles (MP)}

\subsubsection{Morphology, Sizing, and Size Distribution}

CUR nCmP displayed a wrinkled surface with visibly encapsulated NP as seen in Figure 2 and Figure S3. The raisin-like morphology of the $\mathrm{nCmP}$ can be attributed to the early formation of nanoparticle shells in the solution droplets during spray drying, which determines the geometric size of $\mathrm{nCmP}$. As the drying process proceeds, the remaining solvent evaporates from the droplet center, resulting in hollow particles that tend to shrink (Atalar and Dervisoglu, 2015; Gu et al., 2015).

CUR MP were collapsed, wrinkled spheres as seen in Figures 2D-F. Altering the Ac-Dex composition of the particles had no impact on particle morphology. The geometric diameters $(\mathrm{dg})$ of the CUR nCmP and MP systems are shown in Table 2. All of the MP $\mathrm{dg}$ were approximately $1 \mu \mathrm{m}$ in size, which is reported to make the particles vulnerable to macrophage uptake (Sung et al., 2009). In contrast, the NP released from $\mathrm{nCmP}$ systems can escape macrophage clearance upon reaching the deep lung. 


\subsubsection{Analysis of Particle Density}

The density of the particles was determined via tapped density measurements, as shown in Table 2. CUR nCmP exhibited tapped density values around $0.12 \mathrm{~g} / \mathrm{cm}^{3}$, while the MP system showed values around $0.05 \mathrm{~g} / \mathrm{cm}^{3}$. These density values are relatively low compared with the raw materials $\left(\sim 1 \mathrm{~g} / \mathrm{cm}^{3}\right)$, which can be attributed to the wrinkled morphology and hollow structures of the particle systems. It has been reported that particles > $1 \mu \mathrm{m}$ in diameter with greater density will deposit in the lungs by sedimentation (Heyder, 2004). Therefore, the increased density of CUR nCmP system as compared to MP could enhance their rate of deposition into the deep lung.

\subsubsection{Loading and In Vitro Release of CUR}

CUR was successfully encapsulated into both the $\mathrm{nCmP}$ and MP systems as seen

in Table 2. The MP systems prepared via closed mode, organic spray drying exhibited higher encapsulation efficiency (EE, > 50\%) than the $\mathrm{nCmP}$ systems (approximately $30 \%)$ prepared in open mode in aqueous solutions. The lower $\mathrm{EE}$ of the $\mathrm{nCmP}$ can be attributed to the EE of the original CUR-loaded NP, which was also approximately $30 \%$ (Table S1). The spray drying process had no influence on the CUR loading and EE for the $\mathrm{nCmP}$ systems $(\mathrm{p}<0.05)$, which indicates that the drug loading of $\mathrm{nCmP}$ systems can be determined during NP preparation.

Results of the in vitro release of CUR from both $\mathrm{nCmP}$ and MP systems in modified phosphate $(\mathrm{pH}$ 7.4) and acetate $(\mathrm{pH}$ 5) buffers at physiological temperature $\left(37^{\circ} \mathrm{C}\right)$ are reported in Figure 3 as the percentage of cumulative drug released over time.

As shown in Table S2, the particle systems exhibited shorter release durations and 
increased release of CUR $(\mathrm{p}<0.05)$ at acidic $\mathrm{pH}$ with the exception of nCmP-5min, which only exhibited a shorter release duration. These results are in accordance with previous studies (Meenach et al., 2012; Vehring, 2008). The release profiles suggest that the drug will be released at significantly higher rates once the carrier particles reach an acidic environment. This can allow Ac-Dex particles the ability to provide controlled release of a therapeutic payload in cells and tissue with lower $\mathrm{pH}$ values, such as tumor cells and macrophages. In contrast, if the carrier particles remain in the extracellular or neutral $\mathrm{pH}$ environments, the release rate can be reduced, which can minimize systemic and local cytotoxicity (Meenach et al., 2012).

In addition, the $\mathrm{nCmP}$ systems exhibited faster release than the MP systems, which is likely due to the larger surface area available in the nano-sized delivery systems. Upon reaching an aqueous environment, the $\mathrm{nCmP}$ dissociate into nanoparticles with large surface areas and a PVA coating that facilitates particle dispersity, while the MP may agglomerate due to their highly hydrophobic, uncoated surfaces. As a result, the $\mathrm{nCmP}$ systems undergo faster polymer degradation and drug diffusion, resulting in a faster release of payloads than MP at both acidic and physiologic $\mathrm{pH}$.

Particles comprised of Ac-Dex-3h exhibited slower release rates than those comprised of Ac-Dex-5min, indicating that the drug release rate can be controlled by the polymer reaction time. At $\mathrm{pH} 7.4$, particles made of Ac-Dex-h exhibited a drug release rate between Ac-Dex-5 min and Ac-Dex-3h, suggesting that the ratio of different types of Ac-Dex can also act as an important factor in adjusting the release profiles of particle systems. Nevertheless, the drug release rates of the Ac-Dex particles at $\mathrm{pH} 5$ did not follow this trend, which could be explained by one of the following: (1) the release 
profile of Ac-Dex particles is polymer degradation controlled and the decomposition of the Ac-Dex matrix is greatly impacted by the release buffer $\mathrm{pH}$ and (2) the release profile of Ac-Dex particles is both polymer degradation and drug diffusion controlled. In previous studies, drug release from Ac-Dex particles was associated with Ac-Dex degradation (Bachelder et al., 2008; Kauffman et al., 2012; Meenach et al., 2012). However, Ac-Dex degradation may result in the surface erosion of particles, formation of large pores in the particles that facilitate drug diffusion, or both at the same time. As a result, the drug release profile could be controlled by drug diffusion through water-filled pores (diffusion controlled), polymer erosion on the particle surface (erosion controlled), or both drug diffusion and surface erosion (diffusion and erosion controlled), respectively (Broaders et al., 2009).

In order to further illustrate the mechanism of drug release of Ac-Dex particles, we fitted the CUR release data to several commonly utilized drug release models, including: (1) a first order model and (2) Hixson-Crowell model for drug dissolutioncontrolled release, (3) Higuchi model modified to fit burst release at time 0, (4) Korsmeyer-Peppas model and (5) Baker-Lonsdale for drug diffusion-controlled release, (6) Hopfenberg model for surface erosion-controlled release, (7) Baker's model for both degradation and diffusion-controlled release, and (8) Weibull model as a general empirical equation to describe a dissolution or release process (Bohrey et al., 2016; Costa and Sousa Lobo, 2001; Kamaly et al., 2016; Seidlitz and Weitschies, 2012; Shuwisitkul, 2011). The coefficient of determinations $\left(\mathrm{R}^{2}\right)$ of the fit for the models are summarized in Table S3. The modified Higuchi and Baker-Lonsdale models exhibited higher $\mathrm{R}^{2}$ compared with other models, indicating that the drug release profiles of all Ac-Dex 
particles at both acidic and neutral $\mathrm{pH}$ was due primarily to drug diffusion. For Baker's model (Shuwisitkul, 2011) that describes a degradation and diffusion process, the optimal coefficient $\mathrm{k}$ was 0 , thus the equation of Baker's model exhibited the same form as the equation for the Higuchi model. Since the degradation of Ac-Dex was observable during the release experiments, the release profiles of Ac-Dex particles can be explained by the mechanism of drug diffusion through water-filled pores (Kamaly et al., 2016). In the process of drug diffusion through water-filled pores, water was absorbed by Ac-Dex particles and filled in the pores of the polymer matrix, through which the drug diffused into the buffer. As polymer degraded, both pore size and number increased, resulting in enhanced drug release. Therefore, the reaction time of Ac-Dex affected the drug release rate significantly by controlling the formation of pores of particle matrix but not polymer degradation on the surface. Meanwhile, the water absorption into the particles may also influence the drug release rate, which can be supported by the fact that Ac-Dex-3h had a higher ratio of total hydrophobic acetalated group conversion than Ac-Dex-5min. The fitted release curves using modified Higuchi model are shown in Figure S4 along with the original data points. The model was modified to fit the burst release at time 0 of the particle systems, which can be attributed to CUR being initially available on the surface of the particles. The $\mathrm{nCmP}$ systems exhibited a high release at time 0 because the nanoparticle suspension was sonicated before spray drying to form a uniform dispersion, which may cause CUR release in to the suspension.

\subsubsection{Karl Fischer Titration}


The residual water content of CUR nCmP and MP is shown in Table 2. The water content of $\mathrm{nCmP}$ system was approximately $8 \%$, while that of MP system was approximately $6 \%$. The lower water content of MP samples is likely due to the absence of water during the closed mode spray drying process. All particle systems showed acceptable water content for aerosol formulations. In general, reducing the water content in inhalable dry powders can significantly improve their dispersion properties and enhance the stability of the powders during storage (Hickey et al., 2007; Mohammadi et al., 2010). Correspondingly, low water content in inhalable dry powders is highly favorable for efficient dry powder aerosolization and effective particle delivery (Mohammadi et al., 2010; Wu et al., 2013).

\subsubsection{Differential Scanning Calorimetry}

Figure 4 shows DSC thermograms of the raw materials used in particle preparation and the final CUR nCmP and CUR MP systems. Both the raw Ac-Dex-5min and Ac-Dex-3h displayed endothermic phase transition peaks due to melting $\left(\mathrm{T}_{\mathrm{m}}\right)$ near $170{ }^{\circ} \mathrm{C}$. The peaks were broad because of the wide size distribution of Ac-Dex polymer crystallites. None of the CUR nCmP systems exhibited a peak corresponding to Ac-Dex melting, which indicates that the Ac-Dex was transformed in an amorphous state by rapid precipitation during NP formation. The CUR MP systems exhibited broad phase transition peaks near $160{ }^{\circ} \mathrm{C}$, which corresponds to the melting of Ac-Dex. This phase transition shifted to the lower temperature range, indicating a reduction in the crystallinity of Ac-Dex after the spray drying process. 


\subsubsection{Powder X-ray Diffraction (PXRD)}

X-ray diffraction diffractograms of the raw materials, physical mixture of Ac-Dex and CUR, CUR nCmP, and CUR MP are shown in Figure 5. No peaks were present for either raw Ac-Dex samples, suggesting an irregular distribution or lack of Ac-Dex crystallites. The absence of diffraction peaks from Ac-Dex is quite different from commercialized polymers such as PLGA, which exhibits strong XRD characterization peaks (Mohammadi et al., 2010). This phenomenon is likely because the Ac-Dex was collected by rapid precipitation in water, which prevents the formation of large polymer crystallites. Strong peaks were present for raw CUR indicating that it was in crystalline form prior to spray drying. XRD diffractograms of the physical mixture, CUR $\mathrm{nCmP}$ and CUR MP were absent of any diffraction peaks corresponding to raw CUR, which was due to the dilution effect of Ac-Dex. The results obtained from the XRD diffractograms confirmed those from DSC thermograms, which show that raw CUR was converted to amorphous forms during the particle manufacturing process.

\subsubsection{In Vitro Aerosol Dispersion Performance Using Next Generation Impactor (NGI)}

In vitro aerosol dispersion performance properties of the $\mathrm{nCmP}$ were evaluated using a Next Generation Impactor ${ }^{\mathrm{TM}}$ coupled with a human DPI device (Figure 6 and Figure 7). The results indicated that the formulated $\mathrm{nCmP}$ and MP are favorable for efficient dry powder aerosolization and effective targeted pulmonary delivery. The experimental mass mean aerodynamic diameter $\left(\mathrm{MMAD}_{\mathrm{E}}\right)$ values of all particle systems were approximately $2 \mu \mathrm{m}$, while the geometric standard deviation (GSD) values were 2 $3 \mu \mathrm{m}$. The MMADE values were within the range of $1-5 \mu \mathrm{m}$ that is required for 
predominant deposition of particles into the deep lung region (Meenach et al., 2013b), which would be desirable to deliver therapeutics for the treatment of both local and systematic diseases through the lung. The theoretical mass mean aerodynamic diameter (MMADт, Table 2), calculated using the geometric diameter and tapped density, was lower than the experimental MMAD. This discrepancy is likely due to particle agglomeration, which increased the geometric size of the dry powder particulates. All of the particle systems exhibited low tapped density values, which supports the hypothesis that the particles are likely hollow. This can also be attributed to their wrinkled surface morphology, as seen in SEM analysis. The GSD values were within the range of those previously reported and the respirable fraction (RF), fine particle fraction (FPF), and emitted dose (ED) values were all higher than reports from similar systems (Meenach et al., 2013a; Meenach et al., 2013b; Ungaro et al., 2006). The formulated Ac-Dex particle systems are expected to achieve an improved therapeutic effect with a reduced amount of payloads by effectively delivering drugs into the deep lung region.

\section{CONCLUSIONS}

Two types of pulmonary delivery systems were successfully formulated using AcDex with two different degradation rates. The resulting CUR MP were wrinkled spheres (approximately $1 \mu \mathrm{m}$ ), while $\mathrm{nCmP}$ were similar in size with wrinkled surfaces that showed the presence of nanoparticles. The variations in the drug release rates from the Ac-Dex particles were influenced by the Ac-Dex reaction time, ratio of two types of AcDex, and the particle size, which could be easily tuned during the manufacturing process. The $\mathrm{pH}$ value of the environment also had a significant influence on the release profiles, 
allowing the Ac-Dex particles to release the payload in a controlled fashion. All $\mathrm{nCmP}$ and MP systems exhibited desirable properties as dry powder inhalation formulations, including small aerodynamic diameters, which is suitable for deep lung delivery, low water content, which is favorable for particle storage, and amorphization of a crystalline payload, which improves the efficiency of drug dissolution. Overall, the engineered AcDex aerosol particle systems have the potential for targeted delivery of therapeutics into the deep lung.

\section{ACKNOWLEDGEMENTS}

The authors gratefully acknowledge financial support from an Institutional Development Award (IDeA) from the National Institute of General Medical Sciences of the National Institutes of Health under grant number P20GM103430. The content is solely the responsibility of the authors and does not necessarily represent the official views of the National Institutes of Health. This material is based upon work conducted at a Rhode Island NSF EPSCoR research facility, supported in part by the National Science Foundation EPSCoR Cooperative Agreement \#EPS-1004057. In addition, this material is based in part upon work supported by the National Science Foundation under grant number \#1508868. Any opinions, findings, and conclusions or recommendations expressed in this material are those of the authors and do not necessarily reflect the view of the National Science Foundation. Finally, the authors thank RI-INBRE for UPLC access and RIN2 for SEM, DLS, PXRD, and DSC access. 


\section{REFERENCES}

Akl, M.A., Kartal-Hodzic, A., Oksanen, T., Ismael, H.R., Afouna, M.M., Yliperttula, M., Samy, A.M., Viitala, T., 2016. Factorial design formulation optimization and in vitro characterization of curcumin-loaded PLGA nanoparticles for colon delivery. Journal of Drug Delivery Science and Technology 32, Part A, 10-20.

Atalar, I., Dervisoglu, M., 2015. Optimization of spray drying process parameters for kefir powder using response surface methodology. LWT - Food Science and Technology 60, 751-757.

Bachelder, E.M., Beaudette, T.T., Broaders, K.E., Dashe, J., Fréchet, J.M.J., 2008. Acetal-Derivatized Dextran: An Acid-Responsive Biodegradable Material for Therapeutic Applications. Journal of the American Chemical Society 130, 10494-10495. Belotti, S., Rossi, A., Colombo, P., Bettini, R., Rekkas, D., Politis, S., Colombo, G., Balducci, A.G., Buttini, F., 2015. Spray-dried amikacin sulphate powder for inhalation in cystic fibrosis patients: The role of ethanol in particle formation. European Journal of Pharmaceutics and Biopharmaceutics 93, 165-172.

Bohrey, S., Chourasiya, V., Pandey, A., 2016. Polymeric nanoparticles containing diazepam: preparation, optimization, characterization, in-vitro drug release and release kinetic study. Nano Convergence 3, 1-7.

Broaders, K.E., Cohen, J.A., Beaudette, T.T., Bachelder, E.M., Fréchet, J.M.J., 2009. Acetalated dextran is a chemically and biologically tunable material for particulate immunotherapy. Proceedings of the National Academy of Sciences 106, 5497-5502.

Cohen, J.A., Beaudette, T.T., Cohen, J.L., Broaders, K.E., Bachelder, E.M., Frechet, J.M., 2010. Acetal-modified dextran microparticles with controlled degradation kinetics and surface functionality for gene delivery in phagocytic and non-phagocytic cells. Advanced materials (Deerfield Beach, Fla.) 22, 3593-3597.

Collier, M.A., Peine, K.J., Gautam, S., Oghumu, S., Varikuti, S., Borteh, H., Papenfuss, T.L., Sataoskar, A.R., Bachelder, E.M., Ainslie, K.M., 2016. Host-mediated Leishmania donovani treatment using AR-12 encapsulated in acetalated dextran microparticles. Int J Pharm 499, 186-194.

Costa, P., Sousa Lobo, J.M., 2001. Modeling and comparison of dissolution profiles. European Journal of Pharmaceutical Sciences 13, 123-133.

Cui, L., Cohen, J.A., Broaders, K.E., Beaudette, T.T., Frechet, J.M., 2011. Mannosylated dextran nanoparticles: a $\mathrm{pH}$-sensitive system engineered for immunomodulation through mannose targeting. Bioconjugate chemistry 22, 949-957.

Gu, B., Linehan, B., Tseng, Y.-C., 2015. Optimization of the Büchi B-90 spray drying process using central composite design for preparation of solid dispersions. International Journal of Pharmaceutics 491, 208-217. 
He, C., Hu, Y., Yin, L., Tang, C., Yin, C., 2010. Effects of particle size and surface charge on cellular uptake and biodistribution of polymeric nanoparticles. Biomaterials 31 , 3657-3666.

Heyder, J., 2004. Deposition of Inhaled Particles in the Human Respiratory Tract and Consequences for Regional Targeting in Respiratory Drug Delivery. Proceedings of the American Thoracic Society 1, 315-320.

Hickey, A.J., Mansour, H.M., Telko, M.J., Xu, Z., Smyth, H.D., Mulder, T., McLean, R., Langridge, J., Papadopoulos, D., 2007. Physical characterization of component particles included in dry powder inhalers. I. Strategy review and static characteristics. J Pharm Sci 96, 1282-1301.

Hoang, K.V., Borteh, H.M., Rajaram, M.V., Peine, K.J., Curry, H., Collier, M.A., Homsy, M.L., Bachelder, E.M., Gunn, J.S., Schlesinger, L.S., Ainslie, K.M., 2014. Acetalated dextran encapsulated AR-12 as a host-directed therapy to control Salmonella infection. Int J Pharm 477, 334-343.

Jensen, D.M., Cun, D., Maltesen, M.J., Frokjaer, S., Nielsen, H.M., Foged, C., 2010. Spray drying of siRNA-containing PLGA nanoparticles intended for inhalation. Journal of controlled release : official journal of the Controlled Release Society 142, 138-145.

Kamaly, N., Yameen, B., Wu, J., Farokhzad, O.C., 2016. Degradable Controlled-Release Polymers and Polymeric Nanoparticles: Mechanisms of Controlling Drug Release. Chemical Reviews 116, 2602-2663.

Kanthamneni, N., Sharma, S., Meenach, S.A., Billet, B., Zhao, J.-C., Bachelder, E.M., Ainslie, K.M., 2012. Enhanced stability of horseradish peroxidase encapsulated in acetalated dextran microparticles stored outside cold chain conditions. International Journal of Pharmaceutics 431, 101-110.

Kauffman, K.J., Kanthamneni, N., Meenach, S.A., Pierson, B.C., Bachelder, E.M., Ainslie, K.M., 2012. Optimization of rapamycin-loaded acetalated dextran microparticles for immunosuppression. International Journal of Pharmaceutics 422, 356-363.

Kho, K., Cheow, W.S., Lie, R.H., Hadinoto, K., 2010. Aqueous re-dispersibility of spraydried antibiotic-loaded polycaprolactone nanoparticle aggregates for inhaled anti-biofilm therapy. Powder Technology 203, 432-439.

Lai, S.K., Wang, Y.-Y., Hanes, J., 2009. Mucus-penetrating nanoparticles for drug and gene delivery to mucosal tissues. Advanced drug delivery reviews 61, 158-171.

Mansour, H.M., Rhee, Y.-S., Wu, X., 2009. Nanomedicine in pulmonary delivery. International journal of nanomedicine 4, 299-319.

Meenach, S.A., Anderson, K.W., Zach Hilt, J., McGarry, R.C., Mansour, H.M., 2013a. Characterization and aerosol dispersion performance of advanced spray-dried chemotherapeutic PEGylated phospholipid particles for dry powder inhalation delivery in lung cancer. European Journal of Pharmaceutical Sciences 49, 699-711.

Meenach, S.A., Kim, Y.J., Kauffman, K.J., Kanthamneni, N., Bachelder, E.M., Ainslie, K.M., 2012. Synthesis, Optimization, and Characterization of Camptothecin-Loaded Acetalated Dextran Porous Microparticles for Pulmonary Delivery. Molecular Pharmaceutics 9, 290-298.

Meenach, S.A., Vogt, F.G., Anderson, K.W., Hilt, J.Z., McGarry, R.C., Mansour, H.M., 2013b. Design, physicochemical characterization, and optimization of organic solution advanced spray-dried inhalable dipalmitoylphosphatidylcholine (DPPC) and dipalmitoylphosphatidylethanolamine poly(ethylene glycol) (DPPE-PEG) microparticles 
and nanoparticles for targeted respiratory nanomedicine delivery as dry powder inhalation aerosols. International journal of nanomedicine 8, 275-293.

Mohammadi, G., Valizadeh, H., Barzegar-Jalali, M., Lotfipour, F., Adibkia, K., Milani, M., Azhdarzadeh, M., Kiafar, F., Nokhodchi, A., 2010. Development of azithromycinPLGA nanoparticles: physicochemical characterization and antibacterial effect against Salmonella typhi. Colloids and surfaces. B, Biointerfaces 80, 34-39.

Rasband, W.S., 1997-2016. ImageJ.

Seidlitz, A., Weitschies, W., 2012. In-vitro dissolution methods for controlled release parenterals and their applicability to drug-eluting stent testing. The Journal of pharmacy and pharmacology 64, 969-985.

Shuwisitkul, D., 2011. title., Freie Universität Berlin.

Sung, J.C., Padilla, D.J., Garcia-Contreras, L., Verberkmoes, J.L., Durbin, D., Peloquin, C.A., Elbert, K.J., Hickey, A.J., Edwards, D.A., 2009. Formulation and pharmacokinetics of self-assembled rifampicin nanoparticle systems for pulmonary delivery. Pharm Res 26, 1847-1855.

Takashima, Y., Saito, R., Nakajima, A., Oda, M., Kimura, A., Kanazawa, T., Okada, H., 2007. Spray-drying preparation of microparticles containing cationic PLGA nanospheres as gene carriers for avoiding aggregation of nanospheres. Int J Pharm 343, 262-269.

Tomoda, K., Ohkoshi, T., Kawai, Y., Nishiwaki, M., Nakajima, T., Makino, K., 2008. Preparation and properties of inhalable nanocomposite particles: effects of the temperature at a spray-dryer inlet upon the properties of particles. Colloids and surfaces. B, Biointerfaces 61, 138-144.

Ulery, B.D., Nair, L.S., Laurencin, C.T., 2011. Biomedical Applications of Biodegradable Polymers. Journal of polymer science. Part B, Polymer physics 49, 832864.

Ungaro, F., De Rosa, G., Miro, A., Quaglia, F., La Rotonda, M.I., 2006. Cyclodextrins in the production of large porous particles: Development of dry powders for the sustained release of insulin to the lungs. European Journal of Pharmaceutical Sciences 28, 423-432. Vehring, R., 2008. Pharmaceutical Particle Engineering via Spray Drying. Pharm Res 25, 999-1022.

W, F., 2008. The ARLA Respiratory Deposition Calculator.

Wang, Z., Cuddigan, J.L., Gupta, S.K., Meenach, S.A., 2016. Nanocomposite Microparticles (nCmP) for the Delivery of Tacrolimus in the Treatment of Pulmonary Arterial Hypertension. International Journal of Pharmaceutics 512, 305-313.

Wang, Z., Meenach, S.A., 2016. Synthesis and Characterization of Nanocomposite Microparticles (nCmP) for the Treatment of Cystic Fibrosis-Related Infections. Pharmaceutical Research 33, 1862-1872.

Wu, L., Miao, X., Shan, Z., Huang, Y., Li, L., Pan, X., Yao, Q., Li, G., Wu, C., 2014. Studies on the spray dried lactose as carrier for dry powder inhalation. Asian Journal of Pharmaceutical Sciences 9, 336-341.

Wu, X., Zhang, W., Hayes, D., Jr., Mansour, H.M., 2013. Physicochemical characterization and aerosol dispersion performance of organic solution advanced spraydried cyclosporine A multifunctional particles for dry powder inhalation aerosol delivery. Int J Nanomedicine 8, 1269-1283. 


\section{TABLES AND FIGURES}

Table 1. Average diameter (as measured by dynamic light scattering), polydispersity index (PDI), and zeta potential (ZP) of CUR-loaded nanoparticles before spray drying (NP) and after redispersion from nanocomposite microparticles $(\mathrm{nCmP})$ (mean \pm standard deviation, $\mathrm{n}=3$ ).

\begin{tabular}{cccc}
\hline $\begin{array}{c}\text { Particle } \\
\text { System }\end{array}$ & $\begin{array}{c}\text { Average } \\
\text { Diameter }(\mathbf{n m})\end{array}$ & PDI & ZP (mV) \\
\hline NP-5min & $192.2 \pm 2.7$ & $0.07 \pm 0.03$ & $-8.4 \pm 4.1$ \\
NP-h & $201.1 \pm 1.5$ & $0.02 \pm 0.01$ & $-8.0 \pm 3.7$ \\
NP-3h & $206.1 \pm 1.3$ & $0.03 \pm 0.03$ & $-7.0 \pm 1.6$ \\
nCmP-5min & $199.3 \pm 1.3$ & $0.09 \pm 0.02$ & $-14.5 \pm 1.0$ \\
nCmP-h & $210.2 \pm 2.5$ & $0.07 \pm 0.01$ & $-13.3 \pm 1.9$ \\
nCmP-3h & $213.5 \pm 2.4$ & $0.02 \pm 0.00$ & $-11.2 \pm 1.6$ \\
\hline
\end{tabular}

Table 2. Geometric diameter (as measured by SEM imaging and ImageJ analysis), experimental mass median aerodynamic diameter $(\mathrm{MMADE})$, geometric standard deviation (GSD), water content, tapped density, theoretical mean mass aerodynamic diameter (MМАDт), drug loading, and drug encapsulation efficiency (EE) of $\mathrm{nCmP}$ and MP (mean \pm standard deviation, $\mathrm{n}=3$ ).

\begin{tabular}{|c|c|c|c|c|c|c|c|c|}
\hline $\begin{array}{l}\text { Particle } \\
\text { System }\end{array}$ & $\begin{array}{c}\text { Geometric } \\
\text { Diameter } \\
(\mu \mathrm{m})\end{array}$ & $\underset{(\mu \mathrm{m})}{\operatorname{MMAD}_{\mathrm{E}}}$ & $\begin{array}{l}\text { GSD } \\
(\mu \mathrm{m})\end{array}$ & $\begin{array}{c}\text { Water } \\
\text { Content } \\
(\%)\end{array}$ & $\begin{array}{l}\text { Tapped } \\
\text { Density } \\
\left(\mathrm{g} / \mathrm{cm}^{3}\right)\end{array}$ & $\underset{(\mu \mathrm{m})}{\operatorname{MMAD}_{\mathrm{T}}}$ & $\begin{array}{c}\text { Drug } \\
\text { Loading } \\
\text { (mg/100 } \\
\text { mg } \\
\text { particle) }\end{array}$ & $\begin{array}{l}\mathrm{EE} \\
(\%)\end{array}$ \\
\hline $\begin{array}{c}\text { nCmP- } \\
\text { 5min }\end{array}$ & $\begin{array}{c}1.52 \pm \\
0.33\end{array}$ & $\begin{array}{c}1.61 \pm \\
0.16\end{array}$ & $\begin{array}{c}2.37 \pm \\
0.24\end{array}$ & $\begin{array}{c}7.69 \pm \\
0.76\end{array}$ & & & & $\begin{array}{c}28.7 \pm \\
0.32\end{array}$ \\
\hline$\underset{h}{\mathrm{nCmP}-}$ & $\begin{array}{c}1.77 \pm \\
0.46\end{array}$ & $\begin{array}{c}2.05 \pm \\
0.09\end{array}$ & $\begin{array}{c}2.62 \pm \\
0.17\end{array}$ & $\begin{array}{c}7.89 \pm \\
1.56\end{array}$ & & & $\begin{array}{l}0.58 \pm \\
0.008\end{array}$ & $\begin{array}{c}28.4 \pm \\
0.42\end{array}$ \\
\hline$\underset{3 h}{n C m P-}$ & $\begin{array}{c}1.72 \pm \\
0.39\end{array}$ & $\begin{array}{c}1.89 \pm \\
0.09\end{array}$ & $\begin{array}{c}2.70 \pm \\
0.13\end{array}$ & $\begin{array}{l}7.86 \pm \\
0.43\end{array}$ & $\begin{array}{c}0.133 \pm \\
0.002\end{array}$ & $\begin{array}{c}0.64 \pm \\
0.12\end{array}$ & $\begin{array}{l}0.62 \pm \\
0.013\end{array}$ & $\begin{array}{c}31.2 \pm \\
0.63\end{array}$ \\
\hline $\begin{array}{l}\text { MP- } \\
5 \mathrm{~min}\end{array}$ & $\begin{array}{c}0.89 \pm \\
0.30\end{array}$ & $\begin{array}{c}2.38 \pm \\
0.06\end{array}$ & $\begin{array}{c}2.14 \pm \\
0.14\end{array}$ & $\begin{array}{c}6.12 \pm \\
1.33\end{array}$ & $\begin{array}{c}0.050 \pm \\
0.001\end{array}$ & $\begin{array}{c}0.21 \pm \\
0.03\end{array}$ & $\begin{array}{l}1.33 \pm \\
0.092\end{array}$ & $\begin{array}{c}66.3 \pm \\
4.62\end{array}$ \\
\hline MP-h & $\begin{array}{c}1.26 \pm \\
0.41\end{array}$ & $\begin{array}{c}2.21 \pm \\
0.23\end{array}$ & $\begin{array}{c}2.17 \pm \\
0.03\end{array}$ & $\begin{array}{c}5.87 \pm \\
1.85\end{array}$ & $\begin{array}{c}0.050 \pm \\
0.001\end{array}$ & $\begin{array}{c}0.29 \pm \\
0.32\end{array}$ & $\begin{array}{l}1.03 \pm \\
0.030\end{array}$ & $\begin{array}{c}51.6 \pm \\
1.48\end{array}$ \\
\hline MP-3h & $\begin{array}{c}1.05 \pm \\
0.36\end{array}$ & $\begin{array}{c}2.41 \pm \\
0.07\end{array}$ & $\begin{array}{c}2.02 \pm \\
0.08\end{array}$ & $\begin{array}{c}5.23 \pm \\
1.13\end{array}$ & $\begin{array}{c}0.052 \pm \\
0.001\end{array}$ & $\begin{array}{c}0.23 \pm \\
0.03\end{array}$ & $\begin{array}{l}1.12 \pm \\
0.012\end{array}$ & $\begin{array}{c}55.8 \pm \\
0.61\end{array}$ \\
\hline
\end{tabular}



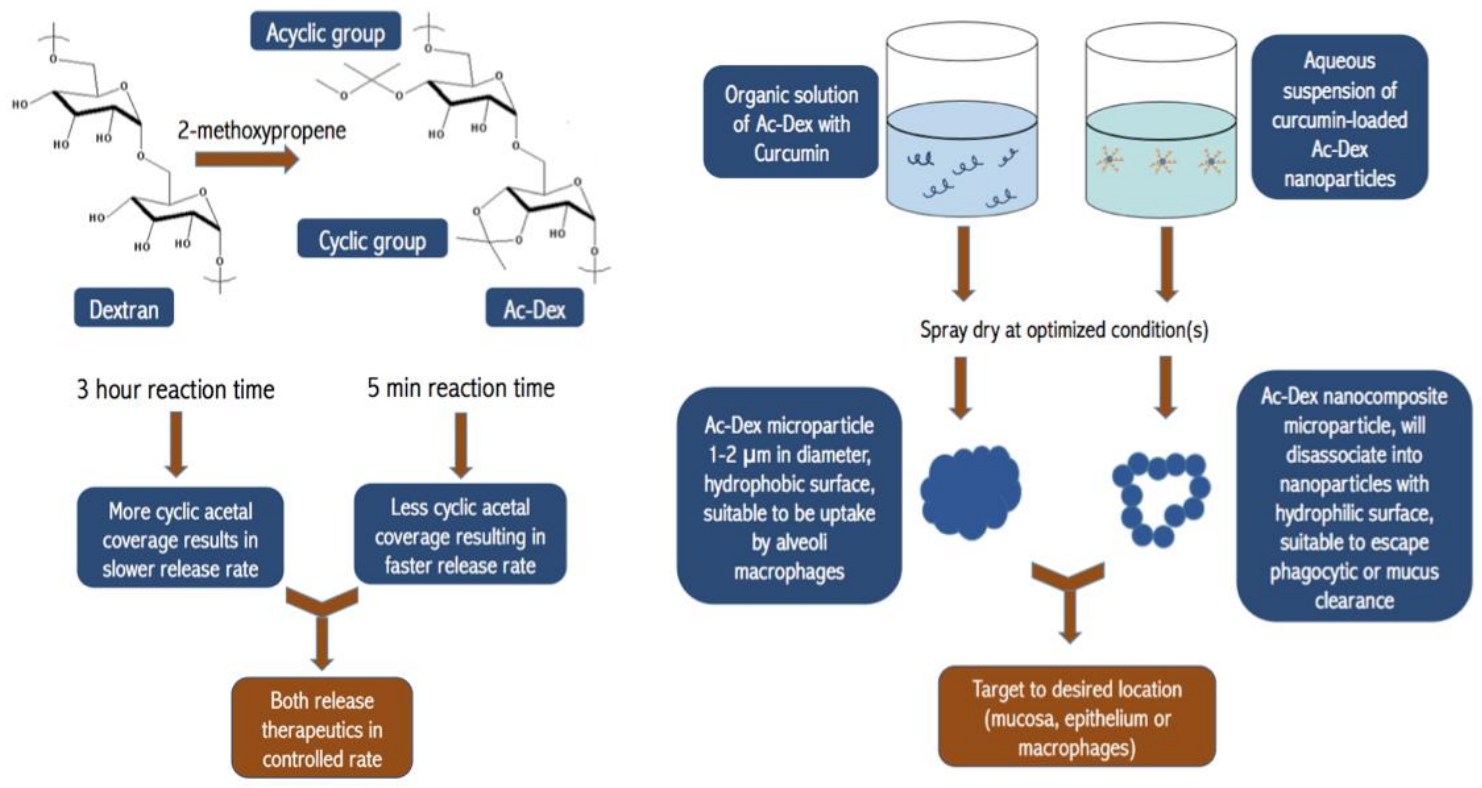

Figure 1. Schematic depicting the synthesis of Ac-Dex (Left) and preparation of nanoparticles and formation of nanocomposite microparticles $(\mathrm{nCmP})$ and microparticles (MP) (Right).

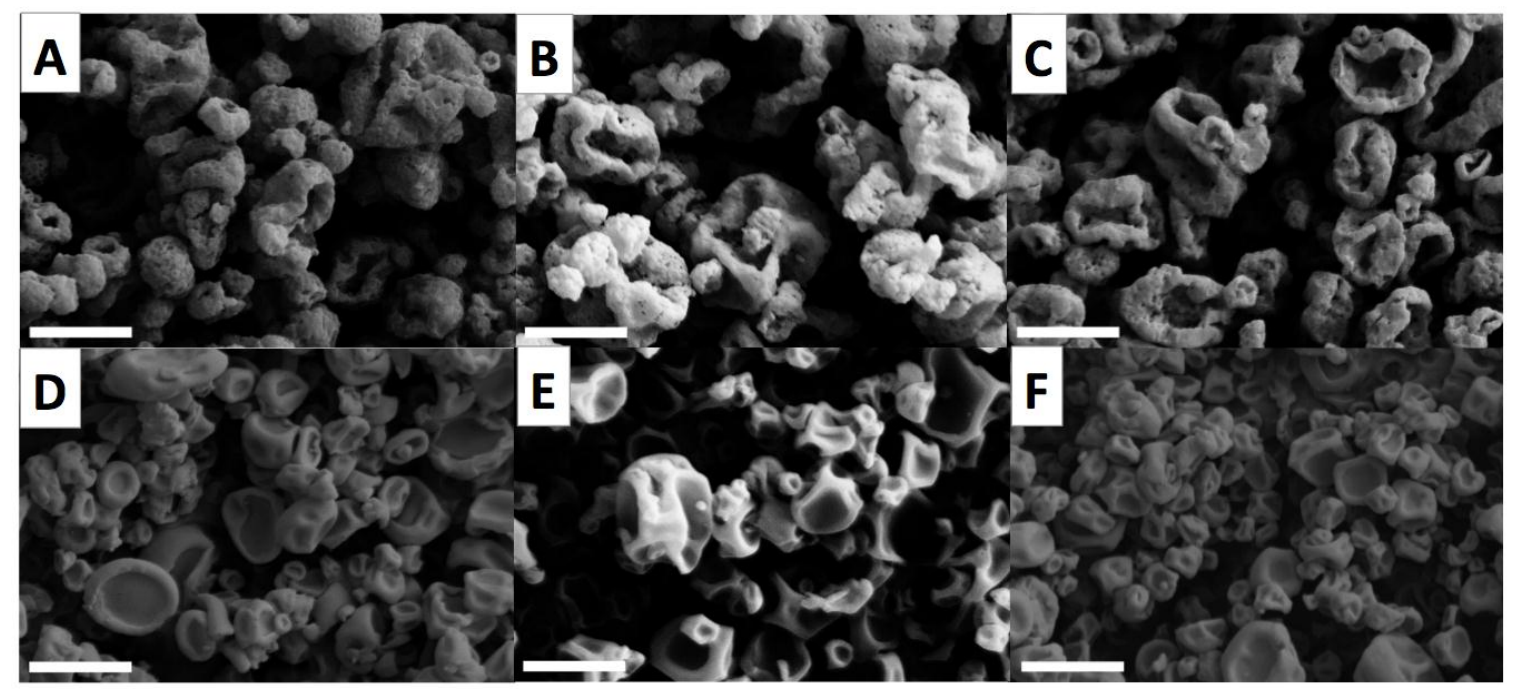

Figure 2. SEM micrographs of curcumin-loaded nanocomposite microparticles (CUR nCmP) and microparticles (CUR MP) including: (A) CUR nCmP-5min, (B) CUR nCmPh, (C) CUR nCmP-3h, (D) CUR MP-5min, (E) CUR MP-h, and (F) CUR MP-3h systems. Scale bar $=2 \mu \mathrm{m}$. 

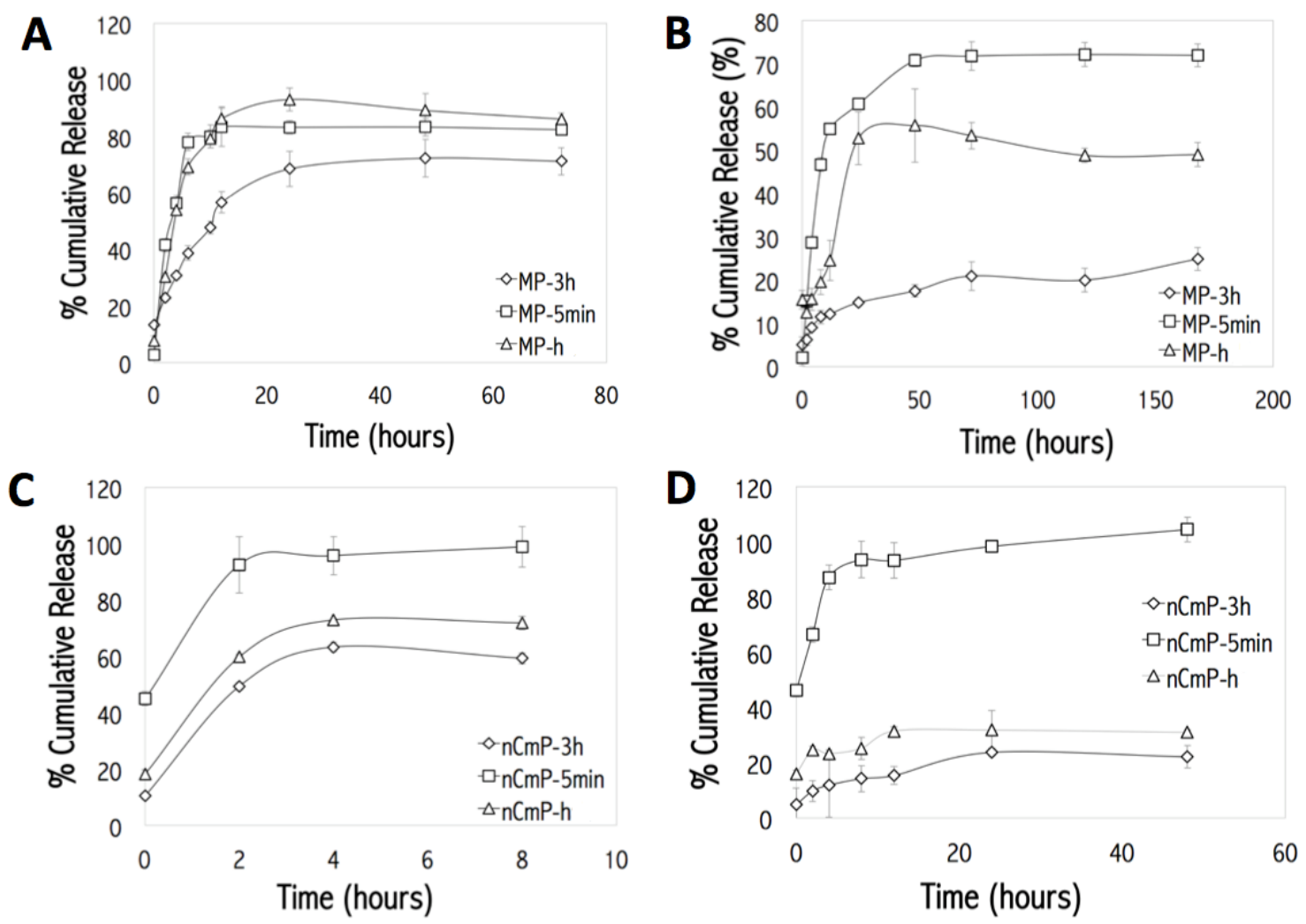

Figure 3. In vitro drug release profiles for curcumin-loaded microparticle (MP) systems at (A) $\mathrm{pH} 5$ and (B) $\mathrm{pH} 7.4$ and curcumin-loaded nanocomposite microparticle (nCmP) system at (C) $\mathrm{pH} 5$ and (D) $\mathrm{pH} 7.4$. 


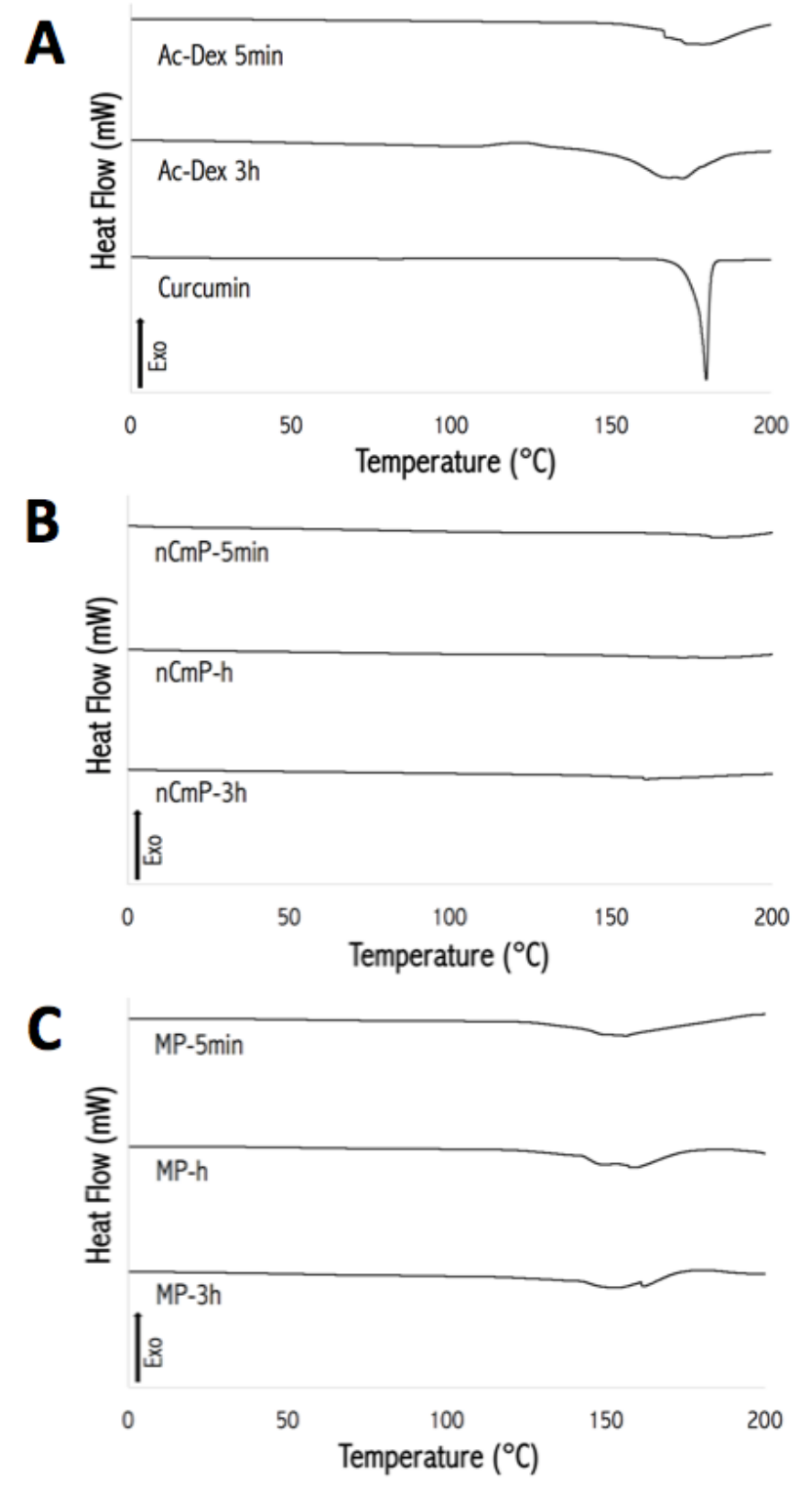

Figure 4. Representative differential scanning calorimetry (DSC) thermograms of (A) raw curcumin (CUR), raw acetalated dextran-5min (Ac-Dex-5min), and raw acetalated dextran-3h (Ac-Dex-3h), (B) CUR nCmP-5min, CUR nCmP-h, and CUR nCmP-3h, and (C) CUR MP-5min, CUR MP-h, and CUR MP-3h. 


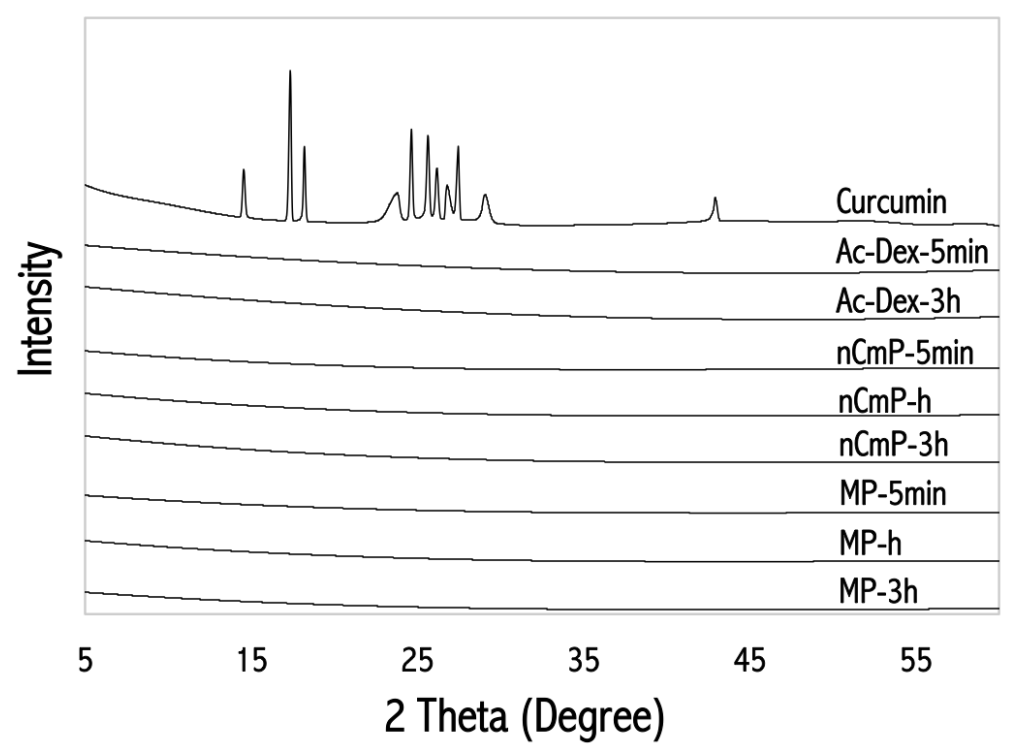

Figure 5. Representative powder X-ray diffractograms (PXRD) of raw curcumin (CUR), raw acetalated dextran-5min (Ac-Dex-5min), raw acetalated dextran-3h (Ac-Dex-3h), CUR nCmP-5min, CUR nCmP-h, CUR nCmP-3h, CUR MP-5min, CUR MP-h, and CUR MP-3h.
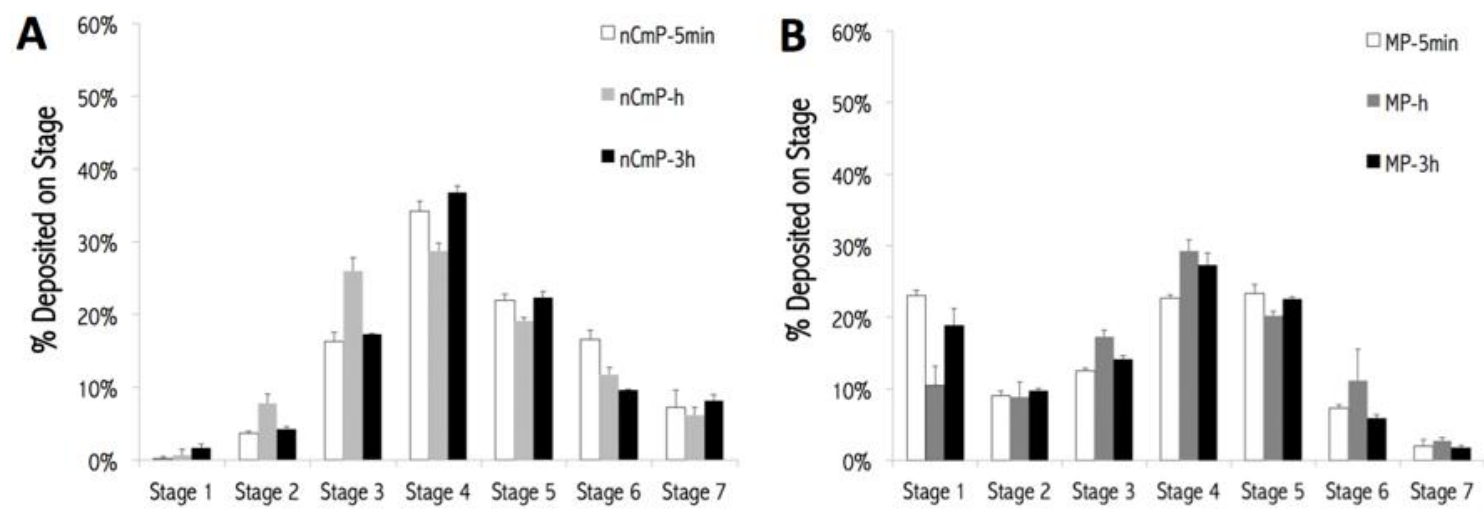

Figure 6. Aerosol dispersion performance of (A) curcumin-loaded nanocomposite microparticles (CUR nCmP) and (B) microparticles (CUR MP) as \% particles deposited on each stage of the Next Generation Impactor ${ }^{\mathrm{TM}}\left(\mathrm{NGI}^{\mathrm{TM}}\right)$. For $\mathrm{Q}=60 \mathrm{~L} / \mathrm{min}$, the effective cutoff diameters (D 50$)$ for each impaction stage are as follows: stage 1 (8.06 $\mu \mathrm{m})$, stage $2(4.46 \mu \mathrm{m})$, stage $3(2.82 \mu \mathrm{m})$, stage $4(1.66 \mu \mathrm{m})$, stage $5(0.94 \mu \mathrm{m})$, stage 6 $(0.55 \mu \mathrm{m})$, and stage $7(0.34 \mu \mathrm{m})($ mean \pm standard deviation, $\mathrm{n}=3)$. 


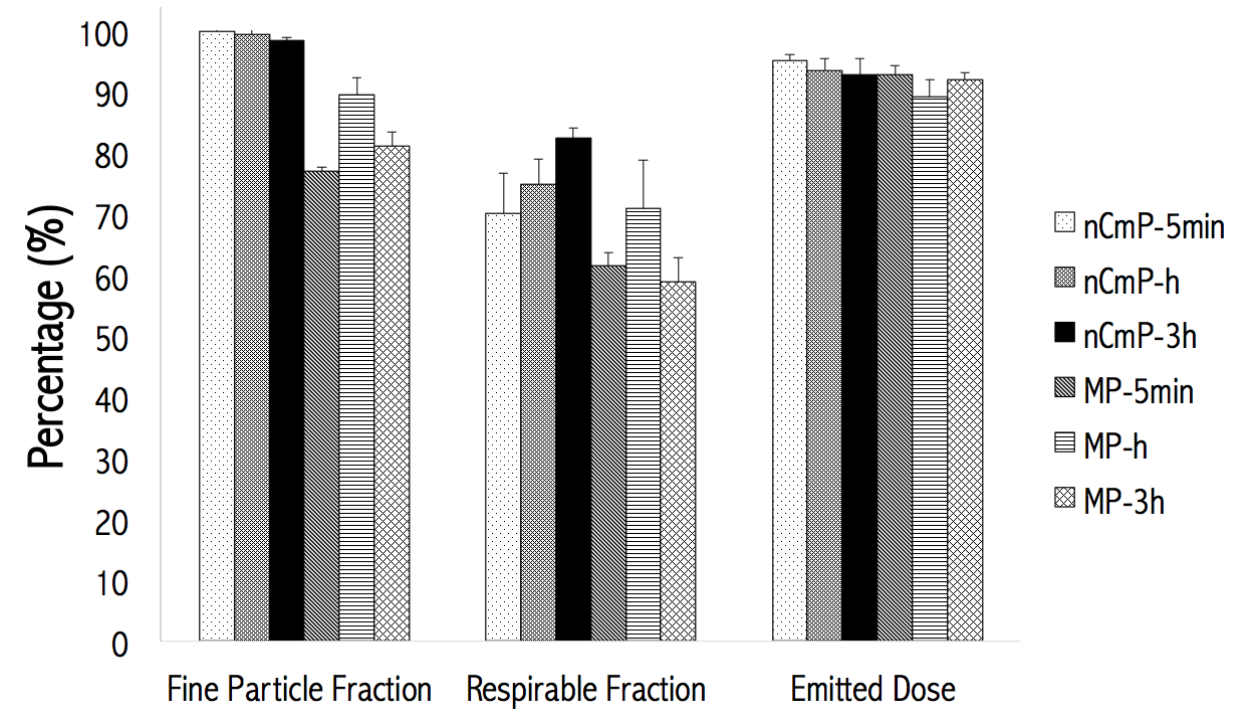

Figure 7. In vitro aerosol dispersion performance properties including fine particle dose (FPD), fine particle fraction (FPF), respirable fraction (RF), and emitted dose (ED) for curcumin loaded nanocomposite microparticles (CUR $\mathrm{nCmP}$ ) and microparticles (CUR MP) (mean \pm standard deviation, $n=3$ ). 


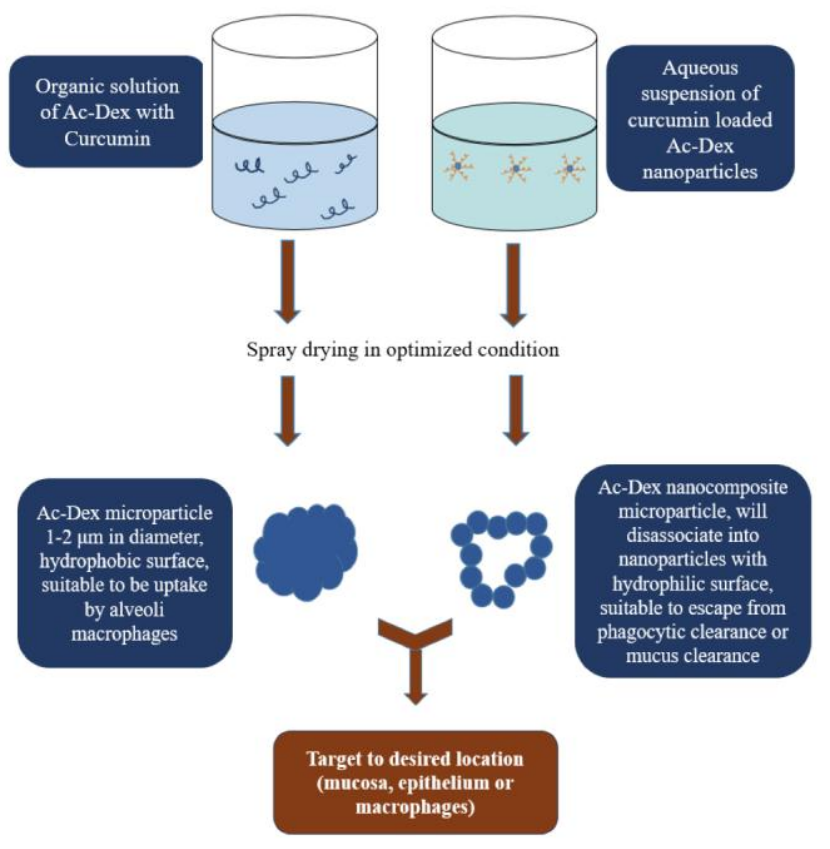

